\author{
Revista Venezolana de Gerencia (RVG) \\ Año 9. № 27, 2004, 391-392 \\ Universidad del Zulia (LUZ) • ISSN 1315-9984
}

\title{
EDITORIAL \\ Salud: Sus tendencias en Venezuela
}

Desde 1999 se registra en Venezuela un proceso de cambio que ha dado paso al desarrollo de nuevas tendencias en materia de política social; particularmente en el ámbito de la salud, se pretende pasar de una política universalista, clientelar y con mediación de los partidos políticos, a una política focalizada, universalista, gratuita, basada en la atención primaria de salud, siendo su población objetivo los sectores más afectados de la población, con ello se busca no solamente la compensación de los mismos sino su inclusión.

Con el nuevo marco institucional, se desea atender la deuda social a través de la aplicación de programas compensatorios en la prestación de servicios de salud, y se ataca el sentido privatista que ha sido puesto en práctica por las gobernaciones venezolanas a partir del proceso de descentralización político territorial, iniciado en la década de los noventa. En este sentido, el Gobierno Nacional adelanta la Misión Barrio Adentro, no sólo como una política que garantiza la atención de manera universal sino como modelo administrativo que busca sustituir los viejos esquemas organizativos.

En la edición de este número, la Revista Venezolana de Gerencia, presenta en su sección En la Mira la Gestión de la Salud en Venezuela, se cuenta en un primer lugar con María Cristina González, y su trabajo"El Género un enfoque transversal en las políticas de salud del Estado Venezolano", cuya discusión esta focalizada hacia uno de los tres ejes principales del Plan Nacional de Desarrollo Económico y Social, además se discute la influencia del patriarcado y las diferencias construidas socialmente, las cuales expresan las inequidades de género en salud. Seguidamente, se presentan dos trabajos que reflejan el estudio de caso a nivel subnacional; uno de ellos es presentado por Belkis Betancourt, Luis Alarcón e Irey Gómez, cuyo título es "Gestión Pública y Salud el caso de la Leishmaniasis en el estado Sucre", este articulo tiene por objeto estudiar el fenómeno salud-enfermedad y la percepción que tienen de ésta los pacientes y no pacientes que acuden al Servicio Dermatológico del estado Sucre (Cumaná-Venezuela); un último artículo de Vilma Vargas y Teresa Gamboa, titulado "Financiamiento a la oferta en Hospitales de Maracaibo: Implicaciones en la demanda de los Servicios", en éste se analizan el origen y volumen del financiamiento hospitalario durante el período 1998-2002, para así observar los cambios en el comportamiento de la demanda de los servicios con relación a la oferta, cobertura poblacional y sus implicaciones en los costos de funcionamiento de dos Hospitales públicos de la ciudad de Maracaibo, Venezuela.

En la sección de Trimestre, se presentan cuatro trabajos, el primero de Haydée Ochoa Henríquez y Yorberth Montes de Oca, titulado"Rendición de Cuentas en la Gestión Pública: Reflexiones Teóricas", cuyo propósito es adelantar algunas reflexiones teóricas sobre el concepto Rendición de Cuentas y contrastarlo con otros conceptos que se han puesto de moda, y a su vez se explora como la Rendición de Cuentas se ha expresado en la práctica bajo ciertos enfoques de 
la administración pública; un segundo articulo de Marta Ochman, "Sociedad Civil y Participación Ciudadana", el mismo propone una delimitación teórica entre el concepto de ciudadanía y de sociedad civil, donde se toman los núcleos semánticos de ambos, asiendo especial referencia a los planteamientos de Haberman y Walter. Se sugiere un uso más restringido de estos conceptos dado que homologarlos no ofrece una solución real. Un tercer trabajo de María Alejandra Ferrer, trata el "Control de Gestión para procesos de apoyo hoteleros", en este se analizan los indicadores para el control de la gestión en hoteles de máxima categoría ubicados en la ciudad de Maracaibo-Venezuela; y por último, el trabajo de José Luis Saavedra, con el titulo"Capital de Marca desde la perspectiva del consumidor", en el cual exploran los conceptos teóricos de Capital de Marca desde la perspectiva del comportamiento que asume el consumidor.

En este número incluimos en la sección Documentos, la exposición presentada por la Dra. Margarita López Maya, con motivo del acto de reconocimiento en la Asamblea Nacional de la ratificación por vía refrendaria del presidente de la República de Venezuela.

\author{
Isabel Rodríguez Colmenares* \\ María T. Rincón Becerra**
}

* Socióloga. Magíster en Ciencia Política. Investigadora del Centro de Estudios de la Empresa (CEE) de la Facultad de Ciencias Económicas y Sociales de La Universidad del Zulia (LUZ). Acreditada en el Programa de Promoción al Investigador (PPI) del FONACIT.

** Lic. en Ciencia Política. Magíster en Dirección y Gestión Pública Local. Investigadora en el CEE de la FCES de LUZ. Becaria del Programa de Investigador Novel (PIN)- FONACIT-LUZ. Acreditada en el PPI del FONACIT. 